\title{
WHOLE PERSON CARE
}

VOLUME $6 \bullet$ NUMBER $2 \bullet 2019 \bullet 1-4$

\section{GUEST EDITORIAL ON BALANCE}

\section{Gordon Crelinsten}

Department of Medicine, Division of Cardiology, Faculty of Medicine, McGill University, Montreal, Quebec, Canada gordon.crelinsten@mcgill.ca

\footnotetext{
W many years, I have been interviewing medical students for residency positions. One of my favourite questions is, "What do you think will be your biggest personal challenge in your first few months of residency training?"
}

Ten years ago, common answers were, "I am concerned that I will not know enough to care for patients. I am worried that I might harm a patient because of my inexperience. I am concerned that I will look over my shoulder for help and there will be no one there. I am concerned about having the responsibility of a physician for the first time."

Today, one of the most common answers is, "I am concerned how I will maintain life-work balance".

There seems to be a subtle shift from emphasis on the patient to the physician. Students now learn that physician wellness is essential to being an effective doctor and that the adage, "physician heal thyself" is of prime importance. Students attend wellness days in medical school where they are encouraged to maintain or develop strategies, techniques and activities to deal with the stress of caring for others as well as time demands inherent in doctoring.

These young people who are carefully chosen to enter medical school are all high achievers. They all have demonstrated academic excellence, while concurrently, according to their autobiographical letters, they have played first violin in the Winnipeg Symphony, have back-packed across the Sahara Desert and have spent their summers meditating on a mountain in Nepal. They are all attentive listeners and 
Guest Editorial

Gordon Crelinsten

have all demonstrated outstanding leadership skills - as captains of competitive teams, presidents of organizations or volunteers in significant helping roles. They have all demonstrated, beginning at a young age, empathy and compassion while being accepting and attentive. They are all fiercely goal oriented and have learned how to sacrifice and to set priorities in order to reach their targets. They have all successfully navigated a difficult journey, have maintained personal strength and integrity while developing skills concentrating on the "other."

What happens during medical training that challenges these attributes? Why do these young people who we hand-pick because of their ability to achieve, maintain high academic rank and enjoy life expanding experiences, express concerns about life-work balance?

Is it our fault? Are we selecting the wrong students? Are we using the wrong criteria? As mentors and role models, are we showing these young people that what they signed on to is not what they find in reality? Are we the ones who have trouble balancing life and work? Do they catch the dis-ease from us?

As I see it, the fulcrum of the life-work see-saw is based on collective features of physicianhood. Both our work activities and life experiences are informed and affected by our "calling." We should not encourage our students to turn off their doctor selves when they are doing other things. I contend that medicine should be viewed as a way of life rather than a means to a lifestyle.

This being said, the potential strain of caring for others in a system which is increasingly complex and difficult to navigate can lead to frustration, dissatisfaction, depression, burnout or a feeling of inadequacy in the face of escalating professional demands.

Medical training places great emphasis on crystallized intelligence, i.e. the ability to call on accumulated facts and know-how to solve problems. However, in real life, situational ambiguity, personal uncertainty and emotional investment require resilient and nimble reactions dependent on skills in priority setting, multi-tasking and managing distractions. These skills with which our bright students have led their lives thus far need to be transferred to the work environment where costs and consequences may be higher because someone else's well-being may be at stake. It is this fluid intelligence that needs to be developed, taught and practiced.

Admittedly, the demands of everyday life often conflict with work obligations. Relationship roles require emotional investment, concentration and often undivided attention. For example, aged parents may need support, husbands, wives and partners may need our time and commitment. Children experience life events which require parental presence. Hobbies need developing; wellness and contentment require effort. 
Guest Editorial

Gordon Crelinsten

How can we do all the above while caring for patients and their problems? When conflicts become unmanageable, when the professional role becomes a burden or when it gets in the way, emotional costs can lead to ineffective behaviors in both realms.

I believe that the ability to be effective in situations that present multiple response alternatives, to perform well when faced with a fast-paced and unpredictable environment entails a skill set which can be learned and practiced.

Contemporary medical practice relies on the smooth functioning of multidisciplinary teams faced with situations requiring dynamic decision making and agile reactions to predictable and unpredictable events. Communication effectiveness and clarity together with strong leadership are essential components of medical practice. During physician training, we should emphasize team scenarios in a simulation centre where safe mistakes and constructive debriefing would enhance the ability to deal with similar situations in real life. These exercises can teach students to quickly assess complex problems, recognize available resources and coordinate responses to achieve optimal results.

These are the same skills needed to deal with the ebb and flow of life-work demands as trainees advance and recede throughout their careers and life trajectories. The ability to manage time and effort, to maximize success at work and still have time to enjoy life can be practiced, taught and developed.

In our medical school, students on clinical rotations are given a "call back" day during which they return to the campus for special lectures and activities. They absent themselves from their ward duties and assigned patients. The students can be expected to deal with this conflict of commitment on their own or they can be offered strategies. For example, they can tell the ward team, "I have a "call back" day tomorrow, I will be absent and return the day after." Or, "I have a "call back" day tomorrow. My three patients have the following tests scheduled and these are the issues I would like you to check up on." Finally, the students whose "call back" day starts at 9:30 am could go to the ward earlier, tell their patients that they will be away, sign out the problems and possibly return after the "call back" day to check on their patients.

I invite the reader to pick the best option and consider whether options should be discussed with students. Perhaps this simple exercise might inform other instances where life and work collide.

Medical school and residency experiences are opportune times to prepare physicians for the stress of conflict resolution, to become comfortable making hard choices and to realize that lifelong experiential learning is part of being a doctor. I contend that this be a cooperative effort. Teachers and learners can work together in a preventive effort to avoid poor outcomes such as addiction, depression, psychological distress or burnout. 
Guest Editorial

Gordon Crelinsten

Clearly, there are individual endeavors that can mitigate the challenges of life-work balance. Periods of quiet reflection using meditation, mindfulness training or spending time in nature have been shown to ease the tension of daily demands.

Moreover, there is strength in shared experiences that is often neglected or under-emphasized. Medical practice, strongly based on knowledge, is highly emotive. The care of and responsibility for others often provoke feelings that cannot be suppressed and thus, call for an outlet.

Maybe, as well as Grand Rounds, we could offer "Autonomic Rounds." Imagine an arena for open discussion between multilevel learners and teachers, a place where feelings of success, failure, happiness, pride, anger or disappointment could be related to clinical events. Participants could share experiences, learn from others and dispel a sense of isolation by showing that the ups and downs of medical practice are universal.

Achieving balance requires effort. To become resilient, i.e. be able to withstand or recover quickly from challenging conditions, compartmentalize, stay in the moment, feel comfortable with ambiguity and uncertainty while doing what is required necessitates awareness and willingness to work with and learn from others. Strong relationships provide a foundation for this effort.

Ultimately, the task of balancing various personal commitments with career demands is a work-inprogress; it begins on day one of medical school and probably never ends. It is a venture demanding continuous attention. It is the realization that life-work boundaries are intertwined and constantly fluctuating.

To prevent overwhelming angst, physicians need to strengthen their ability to reflect on practice and to reflect in practice. Healthy introspection should be emphasized in medical training as well as during one's medical career. We need to reinforce the courage to share feelings. Finally, we should be prepared to make mid-course corrections if and when priorities shift.

We, the mentors and teachers, take these highly motivated, bright young people with precocious life skills into the fold, and somehow, we let them down. Balance need not imply unsteadiness and wavering but rather a comfortable swaying from pleasure to pleasure. 